ANNALES

POLONICI MATHEMATICI

$90.1(2007)$

\title{
A Green's function for $\theta$-incomplete polynomials
}

\author{
by Joe Callaghan (Toronto)
}

\begin{abstract}
Let $K$ be any subset of $\mathbb{C}^{N}$. We define a pluricomplex Green's function $V_{K, \theta}$ for $\theta$-incomplete polynomials. We establish properties of $V_{K, \theta}$ analogous to those of the weighted pluricomplex Green's function. When $K$ is a regular compact subset of $\mathbb{R}^{N}$, we show that every continuous function that can be approximated uniformly on $K$ by $\theta$-incomplete polynomials, must vanish on $K \backslash \operatorname{supp}\left(d d^{c} V_{K, \theta}\right)^{N}$. We prove a version of Siciak's theorem and a comparison theorem for $\theta$-incomplete polynomials. We compute $\operatorname{supp}\left(d d^{c} V_{K, \theta}\right)^{N}$ when $K$ is a compact section.
\end{abstract}

\section{Introduction}

Definition 1.1. For $0<\theta<1$, the set $\pi_{n, \theta}$ shall denote the collection of all polynomials $P$ of the form $P(z)=\sum_{|\alpha|=\lceil n \theta\rceil}^{n} c_{\alpha} z^{\alpha}$. Here $z \in \mathbb{C}^{N}$ and $\lceil x\rceil$ denotes the least integer greater than or equal to $x$. If $P \in \pi_{n, \theta}$ for some $n \geq 0$ then we will refer to $P$ as a $\theta$-incomplete polynomial.

That is, a $\theta$-incomplete polynomial is a polynomial that has no terms of degree smaller than $\theta$ times the degree of the polynomial. For the collection of all polynomials of degree at most $n$ we will simply write $\pi_{n}$. $\theta$-incomplete polynomials of several variables have previously been defined in [2] as polynomials of the form $P(z)=\sum_{|\alpha|=\lfloor n \theta\rfloor}^{n} c_{\alpha} z^{\alpha}$ where $\lfloor x\rfloor$ denotes the integer part of $x$, which yields a slightly different class of polynomials. However, the results in [2] can be verified for the new definition with essentially the same proofs. The new definition is convenient for the purpose of this paper because we would like the function $n^{-1} \log |P|$ to be in $L_{\theta}$ when $P \in \pi_{n, \theta}$. Also, under the new definition the class of $\theta$-incomplete polynomials is closed under multiplication.

By the Weierstrass approximation theorem we know that every continuous function on the closed interval $[0,1]$ can be approximated uniformly by polynomials. The following result of Lorentz, Saff, von Golitschek and

2000 Mathematics Subject Classification: Primary 32U35.

Key words and phrases: pluricomplex Green's function, incomplete polynomials.

Thesis work under the supervision of Professor T. Bloom at the University of Toronto. 
Kuijlaars ([7], [9], [4], [6]) gives a version of the Weierstrass theorem for $\theta$-incomplete polynomials.

Theorem 1.2. For the interval $[0,1] \subset \mathbb{C}$ and $f \in C[0,1]$ there exists $P_{n} \in \pi_{n, \theta}$ with $P_{n} \rightarrow f$ uniformly as $n \rightarrow \infty$ if and only if $f \equiv 0$ on $\left[0, \theta^{2}\right]$.

In other words, on the interval $[0,1]$, uniform limits of $\theta$-incomplete polynomials (in the above sense) are precisely the continuous functions that vanish on the subinterval $\left[0, \theta^{2}\right]$. Given a compact set $K \subset \mathbb{R}^{N}$, let $C(K)$ be the Banach algebra of all continuous functions on $K$ with the supremum norm. Let $C_{\theta}(K)$ be the subalgebra consisting of all functions $f \in C(K)$ admitting uniform approximation $P_{n} \rightarrow f$ as $n \rightarrow \infty$ on $K$ by a sequence of $\theta$-incomplete polynomials $P_{n} \in \pi_{n, \theta}$. Let $Z_{\theta}=\{x \in K: f(x)=0$ for all $f \in$ $\left.C_{\theta}(K)\right\}$. The following result is a consequence of the Stone-Weierstrass theorem.

TheORem $1.3([2])$. For every compact set $K \subset \mathbb{R}^{N} \subset \mathbb{C}^{N}$ we have

$$
C_{\theta}(K)=\left\{f \in C(K): f \equiv 0 \text { on } Z_{\theta}\right\}
$$

For certain sets $K \subset \mathbb{R}^{N}$ that generalize the interval $[0,1]$, the set $Z_{\theta}$ can be determined explicitly to be $\theta^{2} K$ (see [2]).

We make the following definitions, analogous to the Lelong classes $L$ and $L^{+}$and the Siciak extremal function $V_{K}$ (see [10]).

Definition 1.4. For $\theta \in \mathbb{R}$ and $K \subset \mathbb{C}^{N}$, we let

$$
\begin{aligned}
L_{\theta} & :=\left\{u \in L: u(z) \leq \theta \log |z|+C_{u} \text { on } B(0,1)\right\}, \\
L_{\theta}^{+} & :=\left\{u \in L_{\theta}: \max \{\theta \log |z|, \log |z|\}+C_{u} \leq u(z) \text { for all } z \in \mathbb{C}^{N}\right\}, \\
V_{K, \theta}(z) & :=\sup \left\{u(z): u \leq 0 \text { on } K, u \in L_{\theta}\right\} .
\end{aligned}
$$

Here the constant $C_{u}$ depends on the function $u$. Observe that if $P \in \pi_{n, \theta}$ then $n^{-1} \log |P| \in L_{\theta}$. The main purpose of this paper will be to establish the basic properties of $V_{K, \theta}$ analogous to those of $V_{K}$. In particular we prove a version of Siciak's theorem for $\theta$-incomplete polynomials. Then we establish:

THEOREM 1.5. If $K \subset \mathbb{R}^{N}$ is a regular compact set, then $K \backslash S_{\theta} \subset Z_{\theta}$.

Here $S_{\theta}=\operatorname{supp}\left(d d^{c} V_{K, \theta}\right)^{N}$. We will conclude by using this result to compute $S_{\theta}$ for certain subsets of $\mathbb{R}^{n}$. The second section of the paper will review some basic facts from pluripotential theory and weighted pluripotential theory. Many of the results in the third section are similar to results about $V_{K}$ which can be found in [5]. Finally, I would like to thank the referee, who suggested the general statement of Theorem 4.13 and whose comments have improved the overall coherence and quality of this exposition.

2. Background material. We let $\mathbb{C}^{N}$ denote complex $N$-space and we will write $z=\left(z_{1}, \ldots, z_{N}\right)$ where $z_{i} \in \mathbb{C}$ for elements of $\mathbb{C}^{N}$. An $N$-multi- 
index is an $N$-tuple of non-negative integers $\alpha=\left(\alpha_{1}, \ldots, \alpha_{N}\right)$. We will use the multi-index notation $z^{\alpha}$ to denote the monomial $z_{1}^{\alpha_{1}} \cdots z_{N}^{\alpha_{N}}$. The degree of this monomial is $|\alpha|=\alpha_{1}+\cdots+\alpha_{N}$. The open unit ball in $\mathbb{C}^{N}$ is denoted by $B(0,1)$. The Lelong class, the class of logarithmically homogeneous plurisubharmonic functions and the pluricomplex Green function for $K$ are defined respectively as (see [10])

$$
\begin{aligned}
L & :=\left\{u \in \operatorname{PSH}\left(\mathbb{C}^{N}\right): u(z) \leq \log |z|+C_{u} \text { on } \mathbb{C}^{N} \backslash B(0,1)\right\}, \\
H & :=\left\{u \in \operatorname{PSH}\left(\mathbb{C}^{N}\right): u(\lambda z)=u(z)+\log |\lambda| \text { for all } \lambda \in \mathbb{C} \text { and } z \in \mathbb{C}^{N}\right\}, \\
V_{K}(z) & :=\sup \{u(z): u \leq 0 \text { on } K, u \in L\} .
\end{aligned}
$$

For every function $f$ on an open set $U \in \mathbb{C}^{N}$, the upper semicontinuous regularization of $f$ is defined as $f^{*}(z):=\limsup _{w \rightarrow z} f(w)$. A set $P$ is called pluripolar if for every $x \in P$ there is a neighbourhood $U$ of $x$ and $v \in \operatorname{PSH}(U)$ with $v=-\infty$ on $U \cap P$. A set $P$ is called $L$-polar if $P \subset\left\{x \in \mathbb{C}^{N}: v=-\infty\right\}$ for some $v \in L$. It is known from [5] that a set $P$ is pluripolar if and only if it is $L$-polar. A property is said to hold quasi-everywhere (q.e.) if it holds everywhere except possibly on a pluripolar set. A subset $K \subset \mathbb{C}^{N}$ is said to be non-pluripolar in a neighbourhood of any of its points if for every $x \in K$ and for every neighbourhood $U$ of $x$ the intersection $K \cap U$ is non-pluripolar. Let $\|f\|_{K}^{*}:=\inf \left\{\|f\|_{K \backslash P}: P\right.$ a pluripolar subset of $\left.K\right\}$. Note that if $f$ is a continuous function on $K$ and $K$ is non-pluripolar in a neighbourhood of any of its points then $\|f\|_{K}^{*}=\|f\|_{K}$. A compact set $K \subset \mathbb{C}^{N}$ is said to be regular if $V_{K}^{*}=0$ on $K$. We will need the following comparison theorem.

TheOREM $2.1([5])$. Let $G \subset \mathbb{C}^{N}$ be a bounded open set. Suppose that $u$ and $v$ are bounded plurisubharmonic functions on $G$ such that

$$
\liminf _{z \rightarrow w, z \in G}(u(z)-v(z)) \geq 0 \quad \text { for all } w \in \partial G \text {. }
$$

Then

$$
\int_{\{u<v\}}\left(d d^{c} v\right)^{N} \leq \int_{\{u<v\}}\left(d d^{c} u\right)^{N} .
$$

We will also need the following result of Siciak [10].

THEOREM 2.2. If $u \in L$ then there exists a sequence $n_{j} \nearrow \infty$, polynomials $P_{k, j}$ with $\operatorname{deg} P_{k, j} \leq n_{j}$ and integers $t_{j}$ such that $n_{j}^{-1} \max _{1 \leq k \leq t_{j}} \log \left|P_{k, j}(z)\right|$ decreases to $u(z)$ as $j \rightarrow \infty$.

We state the following definitions and results from weighted pluripotential theory for reference, as they are similar to the definitions and results in this paper. A good introduction to weighted pluripotential theory is given in Appendix B of [8].

Definition 2.3. If $K \subset \mathbb{C}^{N}$ is a closed set and $w$ is a non-negative realvalued function on $K$ then $w$ is called a weight function. A weight function 
$w$ is called admissible if

(i) $w$ is upper semicontinuous,

(ii) the set of points in $K$ where $w$ is strictly greater than zero is nonpluripolar,

(iii) if $K$ is unbounded then $\|z\| w(z) \rightarrow 0$ as $\|z\| \rightarrow \infty$ in $K$.

Define $Q(z)=-\log w(z)$. Then the weighted pluricomplex Green function of $K$ with respect to $Q$ is

$$
V_{K, Q}(z):=\sup \{u(z): u \leq Q \text { on } K, u \in L\} .
$$

Let $V_{K, Q}^{*}(z)$ denote its upper semicontinuous regularization. Let $\mu_{w}:=$ $\left(d d^{c} V_{K, Q}^{*}\right)^{N}$ and $S_{w}:=\operatorname{supp} \mu_{w}$.

The following result on weighted approximation appears in [2].

Theorem 2.4. Suppose that $K \subset \mathbb{R}^{N} \subset \mathbb{C}^{N}$ is closed, and that $w$ is a continuous admissible weight on $K$. Then there exists a closed set $Z_{w} \subset K$ such that there exists $P_{n}(z) \in \pi_{n}$ with $w^{n} P_{n}(z) \rightarrow f$ uniformly on $K$ as $n \rightarrow \infty$ if and only if $f \in C(K)$ and $f \equiv 0$ on $Z_{w}$.

The next theorem relates the sets $S_{w}$ and $Z_{w}$.

TheOREM 2.5. If $K \subset \mathbb{R}^{N} \subset \mathbb{C}^{N}$ is closed and non-pluripolar in a neighbourhood of any of its points, and if $w$ is a continuous admissible weight on $K$, then $K \backslash S_{w} \subset Z_{w}$.

Definition 2.6. If $K$ is a closed set and $w$ is an admissible weight on $K$ then

$$
\begin{aligned}
S_{w}^{*} & :=\left\{z \in K: V_{K, Q}^{*}(z) \geq Q(z)\right\}, \\
\Phi_{K, Q}(z) & :=\sup \left\{\left|P_{n}(z)\right|^{1 / n}:\left\|w^{n} P_{n}\right\|_{K} \leq 1, P_{n} \in \pi_{n}, n \geq 1\right\}, \\
\Psi_{K, Q}(z) & :=\sup \left\{\left|P_{n}(z)\right|^{1 / n}:\left\|w^{n} P_{n}\right\|_{K}^{*} \leq 1, P_{n} \in \pi_{n}, n \geq 1\right\} .
\end{aligned}
$$

TheOREM 2.7 ([8]). If $K \subset \mathbb{C}^{N}$ is a closed set and $w$ is an admissible weight on $K$ then $S_{w} \subset S_{w}^{*}$ and $S_{w}$ is non-pluripolar.

If $P \in \pi_{n}$ and $\left|w^{n} P\right| \leq M$ q.e. on $S_{w}$ then $|P| \leq M e^{n V_{K, Q}^{*}}$ on $\mathbb{C}^{N}$.

If $P \in \pi_{n}$ then $\left\|w^{n} P\right\|_{K}^{*}=\left\|w^{n} P\right\|_{S_{w}}^{*}$.

If $S \subset K$ is closed and $\left\|w^{n} P\right\|_{S}^{*}=\left\|w^{n} P\right\|_{K}^{*}$ for all $P \in \pi_{n}$ then $S_{w} \subset S$.

Finally, $V_{K, Q}=\log \Phi_{K, Q}$ and $V_{K, Q}^{*}=\left(\log \Psi_{K, Q}\right)^{*}$.

Let $\Gamma$ be a compact subset of a hyperplane $L \subset \mathbb{R}^{N} \backslash\{0\}$. The set $K:=\{t x: 0 \leq t \leq 1, x \in \Gamma\}$ is called a compact section if it is nonpluripolar in a neighbourhood of any of its points. If the hyperplane is given by the equation $\sum_{j=1}^{N} c_{j} x_{j}=d$ then the associated linear form for $K$ is defined as $l(x)=d^{-1} \sum_{j=1}^{N} c_{j} x_{j}$. For a compact section and an appropriate weight $w$, the zero set $Z_{w}$ and the set $S_{w}$ are known explicitly. 
TheOREM $2.8([2])$. If $K \subset \mathbb{R}^{N} \subset \mathbb{C}^{N}$ is a compact section and $w(x)=$ $l(x)^{\theta /(1-\theta)}$, where $l(x)$ is the linear form associated with $K$ and $0<\theta<1$, then $Z_{w}=\theta^{2} K$ and $S_{w}=\overline{K \backslash \theta^{2} K}$.

3. Basic properties of $V_{K, \theta}$. We will begin by making a simple observation regarding $V_{K, \theta}$ :

$$
\begin{aligned}
V_{K, \theta}(z)= & \sup \left\{u(z): u \leq 0 \text { on } K, u \in L_{\theta}\right\} \\
= & \theta \log |z| \\
& +(1-\theta) \sup \left\{\frac{1}{1-\theta}(u(z)-\theta \log |z|): u \leq 0 \text { on } K, u \in L_{\theta}\right\} \\
\geq & \theta \log |z|+(1-\theta) \sup \left\{v(z): v \in L, v(z) \leq \frac{-\theta}{1-\theta} \log |z| \text { on } K\right\} \\
= & \theta \log |z|+(1-\theta) V_{K, Q}(z)
\end{aligned}
$$

where $Q=-\log w$ and $w=|z|^{\theta /(1-\theta)}$. The inequality can be seen by taking $u(z)=(1-\theta) v(z)+\theta \log |z|$ for a given $v$. When $N=1$ we can take $v=(1-\theta)^{-1}(u(z)-\theta \log |z|)$ for a given $u$, because in one complex variable, the function $u(z)-\theta \log |z|$ is a subharmonic function with a removable singularity at the origin. However, for $N>1$, this function is not necessarily plurisubharmonic. We summarize the above discussion in the following theorem.

THEOREM 3.1. If $K \subset \mathbb{C}^{N}$ is a compact set then $V_{K, \theta}(z) \geq \theta \log |z|+$ $(1-\theta) V_{K, Q}(z)$ where $Q=-\log w$ and $w=|z|^{\theta /(1-\theta)}$. If $N=1$, that is, if $K \subset \mathbb{C}$, then equality holds.

Hence in one complex variable, the function $V_{K, \theta}$ is essentially given by the weighted pluricomplex Green's function for the weight $w=|z|^{\theta /(1-\theta)}$.

For $\theta \leq 0$, we have $L=L_{\theta}$ because in this case the additional condition is redundant.

Proposition 3.2. $L_{1}=H$.

Proof. First suppose that $u \in H$. Then $u(0)=-\infty$ and for any $z \in$ $\mathbb{C}^{N} \backslash\{0\}$ we can write $u(z)=u(z /|z|)+\log |z| \leq\|u\|_{\partial B(0,1)}+\log |z|$ on $\mathbb{C}^{N}$. This shows that $u \in L_{1}$. Conversely, suppose that $u \in L_{1}$. Then on any $\mathbb{C}$-line through the origin the function $v(z)=u(z)-\log |z|$ is subharmonic on $\mathbb{C} \backslash\{0\}$ and is bounded from above. From the removable singularity theorem for subharmonic functions it follows that $v$ extends (on this line) to an entire subharmonic function that is bounded above. Hence $v$ is constant on lines through the origin. The last statement is equivalent to saying that $u \in H$.

Proposition 3.3. For $\theta>1$, we have $L_{\theta}=\{-\infty\}$. 
Proof. Let $u \in L_{\theta}$. As in the above proof, the extension of the function $v$ to any line through the origin must be constant. But clearly, $v(0)=-\infty$. So, $v \equiv-\infty$. Consequently, $u \equiv-\infty$. $V_{K, \theta_{1}}$.

Note that when $\theta_{1} \leq \theta_{2}$ we have $L_{\theta_{2}} \subset L_{\theta_{1}}$ and consequently $V_{K, \theta_{2}} \leq$

EXAmple 3.4. For the unit ball, $V_{\overline{B(0,1)}, \theta}(z)=\max \{\theta \log |z|, \log |z|\}$.

A set $P$ is called $L_{\theta}$-polar if it is contained in the set $\{u \equiv-\infty\}$ for some $u \in L_{\theta}$.

Lemma 3.5. For $0<\theta<1$ and $P \subset \mathbb{C}^{N}, P$ is $L_{\theta}$-polar if and only if $P$ is L-polar.

Proof. Suppose that $P$ is $L$-polar. Take $u \equiv-\infty$ on $P, u \in L$. Then the function $v(z)=\theta \log |z|+(1-\theta) u(z) \in L_{\theta}$ and $v \equiv-\infty$ on $P$. The converse is immediate.

Lemma 3.6. If $E \subset \mathbb{C}^{N}$ is pluripolar then $V_{E, \theta}^{*} \equiv \infty$.

Proof. Take $w \in L_{\theta}, w=-\infty$ on $E$. Then $w+n \leq V_{E, \theta}$ for all $n$. It follows that $V_{E, \theta}=\infty$ except possibly on the pluripolar set $\{w=-\infty\}$. So $V_{E, \theta}^{*} \equiv \infty$.

Lemma 3.7. If $E \subset \mathbb{C}^{N}$ is bounded and non-pluripolar then $V_{E, \theta}^{*} \in L_{\theta}^{+}$.

Proof. Observe that $V_{E, \theta}^{*} \leq V_{E}^{*}$. Because $E$ is non-pluripolar we have $V_{E}^{*} \in L$. Consequently, $V_{E, \theta}^{*} \in L$. To show that $V_{E}^{*} \in L_{\theta}$ observe that $V_{E, \theta}^{*} \leq M$ on $B(0,1)$ for some constant $M$. Let $u$ be in the defining class of $V_{E, \theta}$. Then $\theta^{-1}(u-M)$ is a non-positive plurisubharmonic function on $B(0,1)$ with a logarithmic pole at the origin. Letting $g_{B(0,1)}(z, 0)$ denote the Green function [5] for the unit ball with logarithmic pole at the origin, namely $\log |z|$, we conclude that $\theta^{-1}(u-M) \leq g_{B(0,1)}(z, 0)$ on $B(0,1)$. It follows that $u(z) \leq \theta \log |z|+M$ on $B(0,1)$. Taking the supremum over all such $u$ we conclude that $V_{E, \theta}^{*} \leq \theta \log |z|+M$ on $B(0,1)$. Thus $V_{E, \theta}^{*} \in L_{\theta}$. Letting $|E|:=\sup _{z \in E}|z|$ we conclude that $\max \{\theta \log (|z| /|E|), \log (|z| /|E|)\}$ is a candidate for $V_{E, \theta}$. It follows that $V_{E, \theta}^{*} \in L_{\theta}^{+}$.

Lemma 3.8. If $P \subset \mathbb{C}^{N}$ is pluripolar and $E \subset \mathbb{C}^{N}$ is bounded then $V_{E \backslash P, \theta}^{*}=V_{E, \theta}^{*}$.

Proof. If $P$ is pluripolar then $P$ is $L$-polar and hence $L_{\theta}$-polar. Take $u \in L_{\theta}$ with $u \leq 0$ on $E \backslash P$. Take $v \in L_{\theta}$ with $v \leq 0$ on $E \backslash P$ and $v \equiv-\infty$ on $P$. Then

$$
(1-\varepsilon) u+\varepsilon v \leq V_{E, \theta} \leq V_{E, \theta}^{*}
$$


Letting $\varepsilon$ go to zero we conclude that $u \leq V_{E, \theta}^{*}$ q.e., hence everywhere. Therefore, $V_{E \backslash P, \theta}^{*} \leq V_{E, \theta}^{*}$. The reverse inequality is immediate so the result follows.

Lemma 3.9. If $K_{j}$ are compact sets such that $K_{j} \searrow K$ then $V_{K_{j}, \theta} \nearrow$ $V_{K, \theta}$.

Proof. Let $u \in L_{\theta}, u \leq 0$ on $K$ and $\varepsilon>0$. Then the set $\{u<\varepsilon\}$ is a neighbourhood of $K$, so there exists $j_{0}$ such that whenever $j \geq j_{0}$ we have $K_{j} \subset\{u<\varepsilon\}$. In other words, $u-\varepsilon \leq 0$ on $K_{j}$ for all $j \geq j_{0}$. So, $u-\varepsilon \leq V_{K_{j}, \theta}$ for all $j \geq j_{0}$. It follows that $u-\varepsilon \leq \lim _{j \rightarrow \infty} V_{K_{j}, \theta} \leq V_{K, \theta}$. The result follows by taking the supremum over all such functions $u$.

Lemma 3.10. Let $E \subset \mathbb{C}^{N}$ be bounded and $0<\theta<1$. Then $V_{E, \theta+\varepsilon} \nearrow$ $V_{E, \theta}$ as $\varepsilon \searrow 0$.

Proof. Suppose that $u \in L_{\theta}$, that $u \leq 0$ on $E$ and that $\varepsilon>0$ is so small that $\theta+\varepsilon<1$. Let

$$
v_{\varepsilon}(z):=\frac{1-\theta-\varepsilon}{1-\theta} u(z)+\frac{\varepsilon}{1-\theta} \log \frac{|z|}{|E|} .
$$

Then $v_{\varepsilon} \leq 0$ on $E$. Furthermore,

$$
\begin{aligned}
v_{\varepsilon}(z) & \leq \frac{1-\theta-\varepsilon}{1-\theta}\left(\max \{\theta \log |z|, \log |z|\}+C_{u}\right)+\frac{\varepsilon}{1-\theta} \log \frac{|z|}{|E|} \\
& =\max \{(\theta+\varepsilon) \log |z|, \log |z|\}+\frac{1-\theta-\varepsilon}{1-\theta} C_{u}-\frac{\varepsilon}{1-\theta} \log |E| .
\end{aligned}
$$

So, $v_{\varepsilon}(z) \leq V_{E, \theta+\varepsilon}(z)$. Letting $\varepsilon \searrow 0$, we get $u(z) \leq \liminf _{\varepsilon \rightarrow 0} V_{E, \theta+\varepsilon} \leq$ $\lim \sup _{\varepsilon \rightarrow 0} V_{E, \theta+\varepsilon} \leq V_{E, \theta}$. The result follows by taking the supremum over all such functions $u$.

Lemma 3.11. If $K \subset \mathbb{C}^{N}$ is a regular compact set then $V_{K, \theta}^{*}=V_{K, \theta}$.

Proof. Since $K$ is regular it is non-pluripolar, hence $V_{K, \theta}^{*} \in L_{\theta}$ by Lemma 3.7. Also $V_{K, \theta}^{*} \leq V_{K}^{*}=0$ on $K$. Hence $V_{K, \theta}^{*}$ is in the family defining $V_{K, \theta}$. Consequently, $V_{K, \theta}^{*} \leq V_{K, \theta}$.

We have established that if $E$ is non-pluripolar and bounded then $V_{E, \theta}^{*}$ is in the class $L_{\theta}^{+}$. Consequently, $\left(d d^{c} V_{E, \theta}^{*}\right)^{N}$ is well defined [5].

TheOREM 3.12. If $E \subset \mathbb{C}^{N}$ is bounded and non-pluripolar then $\left(d d^{c} V_{E, \theta}^{*}\right)^{N} \equiv 0$ on $\mathbb{C}^{N} \backslash \bar{E} \cup\{0\}$.

Proof. Let $G \subset \mathbb{C}^{N} \backslash \bar{E} \cup\{0\}$ be a bounded open set. Let $u$ be a plurisubharmonic function on $G$ majorized by $V_{E, \theta}^{*}$ on $\partial G$. Then the function $v(z):=\max \left\{u(z), V_{E, \theta}^{*}(z)\right\}$ on $G$ and $:=V_{E, \theta}^{*}(z)$ elsewhere on $\mathbb{C}^{N}$ has $v \in L_{\theta}$ and $v \leq 0$ q.e. on $E$. So, $v$ is non-positive on $E$ except possibly on a pluripolar set $F$. Take $w \in L_{\theta}$ with $w=-\infty$ on $F$ and $w$ non-positive on $E$. 
Then $(1+\varepsilon)^{-1}(v+\varepsilon w)$ is a candidate for $V_{E, \theta}$. So, $(1+\varepsilon)^{-1}(v+\varepsilon w) \leq V_{E, \theta}^{*}$. Letting $\varepsilon \rightarrow 0$ we conclude that $v \leq V_{E, \theta}^{*}$ q.e. in $G$, hence everywhere in $G$. So, $V_{E, \theta}^{*}$ is a maximal plurisubharmonic function on $\mathbb{C}^{N} \backslash \bar{E} \cup\{0\}$. But on this set, $V_{E, \theta}^{*}$ is locally bounded. Hence, $\left(d d^{c} V_{E, \theta}^{*}\right)^{N} \equiv 0$ on $\mathbb{C}^{N} \backslash \bar{E} \cup\{0\}$.

THEOREM 3.13. If $E \subset \mathbb{C}^{N}$ is non-pluripolar and bounded then for some $\varepsilon>0$ sufficiently small, we have $\left(d d^{c} V_{E, \theta}^{*}\right)^{N} \equiv 0$ on $B(0, \varepsilon) \backslash\{0\}$.

Proof. The set $E$ is non-pluripolar, so $E \backslash B(0, \delta)$ is non-pluripolar for $\delta$ sufficiently small. Fix such a $\delta$. Then $V_{E \backslash B(0, \delta), \theta}^{*}$ has a pole at the origin. This means that there exists $\varepsilon$ such that $0<\varepsilon<\delta$ with $V_{E \backslash B(0, \delta), \theta}^{*} \leq 0$ on $B(0, \varepsilon)$. Now fix such an $\varepsilon$. Then $V_{E \backslash B(0, \varepsilon), \theta}^{*} \leq 0$ on $B(0, \varepsilon)$. Hence, $V_{E \backslash B(0, \varepsilon), \theta}^{*} \leq 0$ q.e. on E. By the same argument used in the preceding proof we conclude that $V_{E \backslash B(0, \varepsilon), \theta}^{*} \leq V_{E, \theta}^{*}$ q.e., hence everywhere. Thus equality holds. So, on $B(0, \varepsilon) \backslash\{0\}$, we have $\left(d d^{c} V_{E, \theta}^{*}\right)^{N}=\left(d d^{c} V_{E \backslash B(0, \varepsilon)}^{*}, \theta\right)^{N} \equiv 0$.

THEOREM 3.14. If $E \subset \mathbb{C}^{N}$ is non-pluripolar and bounded then in some neighbourhood of the origin, $\left(d d^{c} V_{E, \theta}^{*}\right)^{N} \equiv(2 \pi \theta)^{N} \delta_{0}$ where $\delta_{0}$ is the Dirac delta measure.

Proof. By Theorem 3.9 in [3] or Theorem 6.3.5 in [5] we know that $\left(d d^{c} V_{E, \theta}^{*}\right)^{N}=(2 \pi \theta)^{N} \delta_{0}$ at the origin. By the previous theorem we know that $\left(d d^{c} V_{E, \theta}^{*}\right)^{N}=0$ elsewhere near the origin.

The following theorem and proof are exact counterparts of Lemma 6.5 in $[1]$.

TheOREM 3.15. If $u \in L_{\theta}, v \in L_{\theta}{ }^{+}$, and $u \leq v$ holds $\left(d d^{c} v\right)^{N}$-almost everywhere, then $u \leq v$.

Proof. Without loss of generality, we can make the following two assumptions:

(1) $v(z) \geq \theta \log |z|+((1-\theta) / 2) \log 3$ on $B(0,1)$.

(2) $v(z) \geq \frac{1}{2} \log \left(2+|z|^{2}\right)$ on $\mathbb{C}^{N} \backslash B(0,1)$.

Also, because $\max \{\theta \log |z|, \log |z|\}+C \leq v(z)$ on $\mathbb{C}^{N}$, we can replace $u$ with $\max \{u(z), \theta \log |z|+C, \log |z|+C\}$. Now suppose that $u>v$ at some point in $\mathbb{C}^{N}$ (note that this point is not the origin).

Select $\varepsilon, \delta, \eta$ in such a way that $\varepsilon \theta<\eta \theta<\eta \theta+\delta(1-\theta)<\varepsilon, \delta<\varepsilon$ and

$$
S_{\varepsilon, \delta, \eta}:=\left\{z \in \mathbb{C}^{N}: u(z)+\eta \theta \log |z|+\delta \frac{1-\theta}{2} \log \left(2+|z|^{2}\right)>(1+\varepsilon) v(z)\right\}
$$

is non-empty. Then this set must have positive Lebesgue measure. For $|z|$ large enough, we have

$$
u(z)+\eta \theta \log |z|+\delta \frac{1-\theta}{2} \log \left(2+|z|^{2}\right)<(1+\varepsilon) \frac{1}{2} \log \left(2+|z|^{2}\right) \leq(1+\varepsilon) v(z) .
$$


For $|z|$ small enough, we have

$$
\begin{aligned}
u(z)+\eta \theta \log |z|+\delta & \frac{1-\theta}{2} \log \left(2+|z|^{2}\right) \\
& <(1+\varepsilon)\left[\theta \log |z|+\frac{1-\theta}{2} \log 3\right] \leq(1+\varepsilon) v(z) .
\end{aligned}
$$

It follows that $S_{\varepsilon, \delta, \eta}$ is a bounded set that is also bounded away from zero. In other words, $S_{\varepsilon, \delta, \eta} \subset A$ for some set $A$ of the form $A=\left\{z \in \mathbb{C}^{N}\right.$ : $r<|z|<R\}$. The functions $u(z)+\eta \theta \log |z|+\delta((1-\theta) / 2) \log \left(2+|z|^{2}\right)$ and $(1+\varepsilon) v(z)$ are bounded plurisubharmonic functions on $A$, and we have verified that on the boundary of $A$ the condition necessary to apply Theorem 2.1 is satisfied. So,

$$
\begin{aligned}
0 & <\int_{S_{\varepsilon, \delta, \eta}}\left(d d^{c}\left(\delta \frac{1-\theta}{2} \log \left(2+|z|^{2}\right)\right)\right)^{N} \\
& \leq \int_{S_{\varepsilon, \delta, \eta}}\left(d d^{c}\left(u(z)+\eta \theta \log |z|+\delta \frac{1-\theta}{2} \log \left(2+|z|^{2}\right)\right)\right)^{N} \\
& \leq \int_{S_{\varepsilon, \delta, \eta}}(1+\varepsilon)^{N}\left(d d^{c} v\right)^{N}
\end{aligned}
$$

On $S_{\varepsilon, \delta, \eta}$, we have

$$
\begin{aligned}
(1+\varepsilon) v(z) & <u(z)+\eta \theta \log |z|+\delta \frac{1-\theta}{2} \log \left(2+|z|^{2}\right) \\
& \leq v(z)+\eta \theta \log |z|+\delta \frac{1-\theta}{2} \log \left(2+|z|^{2}\right)
\end{aligned}
$$

$\left(d d^{c} v\right)^{N}$-almost everywhere. This implies that on $S_{\varepsilon, \delta, \eta}$, we have

$$
v(z)<\frac{\eta \theta}{\varepsilon} \log |z|+\frac{\delta}{\varepsilon} \frac{1-\theta}{2} \log \left(2+|z|^{2}\right)
$$

$\left(d d^{c} v\right)^{N}$-almost everywhere. But on the unit ball,

$$
v(z) \geq \theta \log |z|+\frac{1-\theta}{2} \log 3 \geq \frac{\eta \theta}{\varepsilon} \log |z|+\frac{\delta}{\varepsilon} \frac{1-\theta}{2} \log \left(2+|z|^{2}\right),
$$

while on the complement of the unit ball we have

$$
v(z) \geq \frac{1}{2} \log \left(2+|z|^{2}\right) \geq \frac{\eta \theta}{\varepsilon} \log |z|+\frac{\delta}{\varepsilon} \frac{1-\theta}{2} \log \left(2+|z|^{2}\right) .
$$

Consequently, $S_{\varepsilon, \delta, \eta}$ is a set of $\left(d d^{c} v\right)^{N}$ measure zero.

This contradicts the integral inequality derived earlier. Thus, $u \leq v$ on $\mathbb{C}^{N}$.

Definition 3.16. Let $K \subset \mathbb{C}^{N}$ be non-pluripolar and bounded. Let $\mu_{\theta}:=\left(d d^{c} V_{K, \theta}^{*}\right)^{N}, S_{\theta}:=\operatorname{supp} \mu_{\theta}$ and $S_{\theta}^{*}:=\left\{z \in K: V_{K, \theta}^{*} \geq 0\right\}$. 
Lemma 3.17. If $K \subset \mathbb{C}^{N}$ is a non-pluripolar compact set then $S_{\theta} \backslash\{0\}$ $\subset S_{\theta}^{*}$, and the set $S_{\theta} \backslash\{0\}$ is non-pluripolar in a neighbourhood of any of its points.

Proof. We will prove the first assertion by contradiction so suppose that $x_{0} \notin S_{\theta}^{*}$ and $x_{0} \in S_{\theta} \backslash\{0\}$. Then $V_{K, \theta}^{*}\left(x_{0}\right)<0$. Next, $V_{K, \theta}^{*}=V_{K \backslash\left\{x_{0}\right\}, \theta}^{*}$. Let $v=\lim _{\varepsilon \rightarrow 0} V_{K \backslash B\left(x_{0}, \varepsilon\right), \theta}^{*}$. It is easy to see that $L_{\theta}$ is closed under limits of decreasing sequences, so $v \in L_{\theta}$. Furthermore, $v \leq 0$ q.e. on $K \backslash\left\{x_{0}\right\}$. So by the standard argument, $V_{K \backslash\left\{x_{0}\right\}, \theta}^{*} \geq v$. For each $\varepsilon>0$, we have $V_{K \backslash B\left(x_{0}, \varepsilon\right), \theta}^{*} \geq V_{K, \theta}^{*}$. Hence $v \geq V_{K, \theta}^{*}$. So, $v=V_{K, \theta}^{*}$. So $V_{K \backslash B(0, \varepsilon), \theta}^{*}\left(x_{0}\right)<0$ for some $\varepsilon>0$. It follows that $V_{K \backslash B\left(x_{0}, \varepsilon\right), \theta}^{*}<0$ on some $B\left(x_{0}, \delta\right)$ where $0<\delta<$ $\varepsilon$. Then $V_{K \backslash B\left(x_{0}, \delta\right), \theta}^{*}<0$ on $B\left(x_{0}, \delta\right)$. We conclude that $V_{K, \theta}^{*} \geq V_{K \backslash B\left(x_{0}, \delta\right), \theta}^{*}$. The opposite inequality is immediate so it follows that $V_{K, \theta}^{*}=V_{K \backslash B\left(x_{0}, \delta\right), \theta}^{*}$. Hence, $\left(d d^{c} V_{K, \theta}^{*}\right)^{N} \equiv 0$ on $B\left(x_{0}, \delta\right)$. Therefore, $x_{0}$ is not contained in $S_{\theta}$. To see the second assertion, note that on $\mathbb{C}^{N} \backslash\{0\}, V_{K, \theta}^{*}$ is a locally bounded plurisubharmonic function. Hence, $\mu_{\theta}$ places no mass on pluripolar subsets of $\mathbb{C}^{N} \backslash\{0\}$. By the definition of support of a measure, $\mu_{\theta}$ must have positive mass in any neighbourhood of any point of $S_{\theta}$.

REMARK 3.18. Because $V_{K, \theta}=V_{K, \theta}^{*}$ q.e. on $\mathbb{C}^{N}$ it follows that $V_{K, \theta}^{*} \leq 0$ q.e. on $K$ and that $V_{K, \theta}=0$ q.e. on $S_{\theta}^{*}$.

\section{Incomplete polynomials and approximation}

THEOREM 4.1. If $P(z)=\sum_{|\alpha|=\lceil n \theta\rceil}^{n} c_{\alpha, n} z^{\alpha}$ is a $\theta$-incomplete polynomial and $|P(z)| \leq M$ q.e. on $S_{\theta}$ then $|P(z)| \leq M e^{n V_{K, \theta}^{*}}$ on $\mathbb{C}^{N}$.

Proof. Observe that

$$
\frac{1}{n} \log \frac{|P(z)|}{M} \leq 0 \quad \text { q.e. on } S_{\theta} .
$$

Consequently,

$$
\frac{1}{n} \log \frac{|P(z)|}{M} \leq V_{K, \theta}^{*} \quad \text { q.e. on } S_{\theta} .
$$

By Theorem 3.15, this inequality must hold on all of $\mathbb{C}^{N}$.

THEOREM 4.2. If $P(z)=\sum_{|\alpha|=\lceil n \theta\rceil}^{n} c_{\alpha, n} z^{\alpha}$ is a $\theta$-incomplete polynomial then $\|P\|_{K}^{*}=\|P\|_{S_{\theta}}^{*}$.

Proof. We have $|P(z)| \leq\|P\|_{S_{\theta}}^{*}$ q.e. on $S_{\theta}$. Therefore, $|P(z)| \leq$ $\|P\|_{S_{\theta}}^{*} e^{n V_{K, \theta}^{*}}$ on $\mathbb{C}^{N}$. Consequently, $|P(z)| \leq\|P\|_{S_{\theta}}^{*}$ q.e. on $K$. Hence, $\|P\|_{K}^{*} \leq\|P\|_{S_{\theta}}^{*}$. So equality holds. 
Definition 4.3. For $K \subset \mathbb{C}^{N}$ a compact set, let

$$
\begin{aligned}
& \Phi_{K, \theta}(z):=\sup \left\{\left|P_{n}(z)\right|^{1 / n}:\left\|P_{n}\right\|_{K} \leq 1, n \geq 1, P_{n} \in \pi_{n, \theta}\right\}, \\
& \Psi_{K, \theta}(z):=\sup \left\{\left|P_{n}(z)\right|^{1 / n}:\left\|P_{n}\right\|_{K}^{*} \leq 1, n \geq 1, P_{n} \in \pi_{n, \theta}\right\} .
\end{aligned}
$$

The cases $\theta=0$ and $\theta=1$ of the following theorem are proved in [10].

ThEOREM 4.4. For $K \subset \mathbb{C}^{N}$ compact, we have $V_{K, \theta}=\log \phi_{K, \theta}$.

Proof. Let $\varepsilon>0$. Suppose that $u \in L_{\theta+\varepsilon}$ and that $u \leq 0$ on $K$. By Theorem 2.2 we have

$$
u(z)=\lim _{j \rightarrow \infty} \frac{1}{n_{j}} \max _{1 \leq k \leq t_{j}} \log \left|P_{k, j}(z)\right|,
$$

where the sequence is decreasing and each $P_{k, j}$ is a polynomial of degree at most $n_{j}$.

Write $P_{k, j}(z):=\sum_{|\alpha|=0}^{n_{j}} c_{\alpha, k, j} z^{\alpha}$ and let $P_{k, j}^{\prime}(z):=\sum_{|\alpha|=0}^{\left\lfloor n_{j} \theta\right\rfloor} c_{\alpha, k, j} z^{\alpha}$, where $\lfloor x\rfloor$ is the greatest integer that is less than or equal to $x$. That is, $P_{k, j}^{\prime}(z)$ is the part of $P_{k, j}(z)$ consisting of monomials of degree smaller than or equal to $\left\lfloor n_{j} \theta\right\rfloor$. Now,

$$
\begin{aligned}
\left\|P_{k, j}^{\prime}(z)\right\| & \leq\left(\begin{array}{c}
n_{j}+N \\
N
\end{array}\right)\left(\max _{0 \leq|\alpha| \leq\left\lfloor n_{j} \theta\right\rfloor}\left|c_{\alpha, k, j}\right|\right)\left(\max _{0 \leq|\alpha| \leq n_{j}}\left|z_{1}\right|^{\alpha_{1}} \ldots\left|z_{N}\right|^{\alpha_{N}}\right) \\
& \leq\left(\begin{array}{c}
n_{j}+N \\
N
\end{array}\right)\left(\max _{0 \leq|\alpha| \leq\left\lfloor n_{j} \theta\right\rfloor}\left|c_{\alpha, k, j}\right|\right)\left(\max \left\{1,\left|z_{1}\right|, \ldots,\left|z_{N}\right|\right\}\right)^{n_{j}} .
\end{aligned}
$$

By Cauchy's estimate, for any $R<1$ we have

$$
\max _{0 \leq|\alpha| \leq\left\lfloor n_{j} \theta\right\rfloor}\left|c_{\alpha, k, j}\right| \leq \max _{0 \leq|\alpha| \leq\left\lfloor n_{j} \theta\right\rfloor} \frac{\left\|P_{k, j}\right\|_{P(0, R)}}{R^{|\alpha|}} \leq \frac{\left\|P_{k, j}\right\|_{P(0, R)}}{R^{\left\lfloor n_{j} \theta\right\rfloor}} .
$$

Moreover, $u(z) \leq(\theta+\varepsilon) \log |z|+M$ on $B(0,1)$. So, $u(z) \leq(\theta+\varepsilon) \log N^{1 / 2} R$ $+M$ on the Shilov boundary of the polydisc of radius $R$ centered at the origin denoted by $\partial_{S} P(0, R)=\left\{\left(z_{1}, \ldots, z_{N}\right):\left|z_{1}\right|=\cdots=\left|z_{N}\right|=R\right\}$. By Dini's theorem there exists $j_{0}$ such that whenever $j \geq j_{0}$ we have

$$
\frac{1}{n_{j}} \max _{1 \leq k \leq t_{j}} \log \left|P_{k, j}(z)\right| \leq(\theta+\varepsilon) \log N^{1 / 2} R+M+1 \quad \text { on } \partial_{S} P(0, R) .
$$

Hence, $\left\|P_{k, j}\right\|_{P(0, R)}^{1 / n_{j}}=\left\|P_{k, j}\right\|_{\partial_{S} P(0, R)}^{1 / n_{j}} \leq\left(N^{1 / 2} R\right)^{\theta+\varepsilon} e^{M+1}$. Therefore, for all $z \in \mathbb{C}^{N}$ we have

$$
\left|P_{k, j}^{\prime}(z)\right| \leq\left(\begin{array}{c}
n_{j}+N \\
N
\end{array}\right) \frac{\left(\max \left\{1,\left|z_{1}\right|, \ldots,\left|z_{N}\right|\right\}\right)^{n_{j}}}{\left.R^{\left\lfloor n_{j}\right.} \theta\right\rfloor}\left(\left(N^{1 / 2} R\right)^{\theta+\varepsilon} e^{M+1}\right)^{n_{j}} .
$$

Hence,

$$
\limsup _{j \rightarrow \infty} \max _{1 \leq k \leq t_{j}}\left|P_{k, j}^{\prime}(z)\right|^{1 / n_{j}} \leq \frac{N^{1 / 2} R^{\theta+\varepsilon} e^{M+1}\left(\max \left\{1,\left|z_{1}\right|, \ldots,\left|z_{N}\right|\right\}\right)}{R^{\theta}} .
$$


Since this holds for any $1>R>0$ sufficiently small, we have

$$
\limsup _{j \rightarrow \infty} \max _{1 \leq k \leq t_{j}}\left|P_{k, j}^{\prime}(z)\right|^{1 / n_{j}}=0 \quad \text { at every } z \in \mathbb{C}^{N} .
$$

Applying the triangle inequality repeatedly, we get

$$
\begin{aligned}
\limsup _{j \rightarrow \infty} \max _{1 \leq k \leq t_{j}}\left|P_{k, j}(z)-P_{k, j}^{\prime}(z)\right|^{1 / n_{j}} \\
\quad \leq \limsup _{j \rightarrow \infty} \max _{1 \leq k \leq t_{j}}\left(\left|P_{k, j}\right|+\left|P_{k, j}^{\prime}\right|\right)^{1 / n_{j}} \\
\quad \leq \limsup _{j \rightarrow \infty} \max _{1 \leq k \leq t_{j}}\left(\left|P_{k, j}(z)\right|^{1 / n_{j}}+\left|P_{k, j}^{\prime}(z)\right|^{1 / n_{j}}\right)=\lim _{j \rightarrow \infty} \max _{1 \leq k \leq t_{j}}\left|P_{k, j}(z)\right|^{1 / n_{j}} \\
\quad \leq \liminf _{j \rightarrow \infty} \max _{1 \leq k \leq t_{j}}\left(\left|P_{k, j}(z)-P_{k, j}^{\prime}(z)\right|^{1 / n_{j}}+\left|P_{k, j}^{\prime}(z)\right|^{1 / n_{j}}\right) \\
\quad=\liminf _{j \rightarrow \infty} \max _{1 \leq k \leq t_{j}}\left|P_{k, j}(z)-P_{k, j}^{\prime}(z)\right|^{1 / n_{j}}
\end{aligned}
$$

This shows that

$$
u(z)=\lim _{j \rightarrow \infty} \frac{1}{n_{j}} \max _{1 \leq k \leq t_{j}} \log \left|P_{k, j}-P_{k, j}^{\prime}\right| .
$$

We have expressed $u$ as a pointwise limit using $\theta$-incomplete polynomials. However, unlike the original sequence, this sequence is not necessarily decreasing. So instead of Dini's theorem we use Hartogs' lemma. The new sequence can readily be seen to be uniformly bounded on any ball centered at the origin just by applying Cauchy estimates and the fact that the original sequence is uniformly bounded on $P(0,1)$.

Hence, Hartogs' lemma gives us a $j_{1}$ such that whenever $j \geq j_{1}$ we have

$$
\frac{1}{n_{j}} \max _{1 \leq k \leq t_{j}} \log \left|P_{k, j}-P_{k, j}^{\prime}\right| \leq \varepsilon_{1} \quad \text { on } K \text {. }
$$

It follows that

$$
\frac{1}{n_{j}} \max _{1 \leq k \leq t_{j}} \log \left|P_{k, j}-P_{k, j}^{\prime}\right| e^{-\varepsilon_{1} n_{j}} \leq \log \Phi_{K, \theta} .
$$

Thus, $u(z)-\varepsilon_{1} \leq \log \Phi_{K, \theta}(z)$. So, $u(z) \leq \log \Phi_{K, \theta}$. Taking the supremum over all such $u$, we infer that $V_{K, \theta+\varepsilon} \leq \log \Phi_{K, \theta}$. Letting $\varepsilon \rightarrow 0$ we obtain $V_{K, \theta} \leq \log \Phi_{K, \theta}$. The reverse inequality follows from the definition.

Corollary 4.5. If $K \subset \mathbb{C}^{N}$ is a compact set and $0<\theta<1$ then the function $V_{K, \theta}$ is lower semicontinuous on $\mathbb{C}^{N} \backslash\{0\}$.

Proof. We see that $\Phi_{K, \theta}$ is lower semicontinuous since it is a supremum over a family of continuous functions. The result follows from the theorem since the logarithm of a non-negative lower semicontinuous function is lower semicontinuous wherever the function is non-zero. Now, $V_{K, \theta} \geq \log (|z| /|K|)$ on $\mathbb{C}^{N}$ so $\Phi_{K, \theta}$ is non-zero on $\mathbb{C}^{N} \backslash\{0\}$. 
COROLlaRY 4.6. If $K \subset \mathbb{C}^{N}$ is a regular compact set then $V_{K, \theta}$ is a plurisubharmonic function that is continuous on $\mathbb{C}^{N} \backslash\{0\}$.

Corollary 4.7. For $K \subset \mathbb{C}^{N}$ compact, we have $\left(\log \Psi_{K, \theta}\right)^{*}=V_{K, \theta}^{*}$.

Proof. By the definitions, $\Phi_{K, \theta} \leq \Psi_{K, \theta}$. It follows that $V_{K, \theta}^{*} \leq\left(\log \Psi_{K, \theta}\right)^{*}$. For the reverse inequality, let $P_{n}$ be any polynomial of degree at most $n$ from the defining family for $\Psi_{K, \theta}$. Then, $n^{-1} \log \left|P_{n}\right| \leq 0$ q.e. on $K$. So, $n^{-1} \log \left|P_{n}\right| \leq V_{K, \theta}^{*}$ q.e. on $S_{\theta}$. Hence, $n^{-1} \log \left|P_{n}\right| \leq V_{K, \theta}^{*}$ on all of $\mathbb{C}^{N}$. Therefore, $\log \Psi_{K, \theta} \leq V_{K, \theta}^{*}$.

THEOREM 4.8. If $S \subset K \subset \mathbb{C}^{N}$ are compact sets with the property that $\|P\|_{S}^{*}=\|P\|_{K}^{*}$ for all $\theta$-incomplete polynomials $P$ then $S_{\theta} \subset S \cup\{0\}$.

Proof. From the previous corollary we conclude that $V_{S, \theta}^{*}=V_{K, \theta}^{*}$. So, $S_{\theta}=\operatorname{supp}\left(d d^{c} V_{K, \theta}^{*}\right)^{N}=\operatorname{supp}\left(d d^{c} V_{S, \theta}^{*}\right)^{N} \subset S \cup\{0\}$.

THEOREM 4.9. Suppose that $K$ is non-pluripolar and bounded and that a sequence $\left\{P_{n}\right\} \in \pi_{n, \theta}$ converges uniformly on $S_{\theta}$ as $n \rightarrow \infty$. Then $\left|P_{n}\left(z_{0}\right)\right| \rightarrow 0$ as $n \rightarrow \infty$ for all $z_{0} \in\left\{z \in \mathbb{C}^{N}: V_{K, \theta}^{*}(z)<0\right\}$.

Proof. Let $z_{0} \in\left\{V_{K, \theta}^{*}<0\right\}$. Then $\left|P_{n}\left(z_{0}\right)\right| \leq\left\|P_{n}\right\|_{S_{\theta}}^{*} e^{n V_{K, \theta}^{*}\left(z_{0}\right)}$. Consequently, $\left|P_{n}\left(z_{0}\right)\right| \rightarrow 0$ as $n \rightarrow \infty$.

The following result is a counterpart of Theorem 3.2 in [2]. Its proof will require the Stone-Weierstrass theorem, in the following form:

THEOREM 4.10. Let $X$ be a compact Hausdorff space and let $C(X):=$ $\{f: X \rightarrow \mathbb{R}, f$ continuous $\}$. Let $A \subset C(X)$ and $Z(A):=\{x \in X: f(x)=0$ for all $f \in A\}$. Suppose further that $A$ has the following four properties:

(i) closedness under addition and real scalar multiplication,

(ii) closedness under multiplication,

(iii) closedness under uniform limits,

(iv) for all $x_{1} \neq x_{2}$ in $X \backslash Z(A)$ there exists $f \in A$ with $f\left(x_{1}\right) \neq f\left(x_{2}\right)$. Then $A=\{f \in C(X): f \equiv 0$ on $Z(A)\}$.

TheOREM 4.11. Let $K \subset \mathbb{R}^{N} \subset \mathbb{C}^{N}$ be a compact set that is nonpluripolar in a neighbourhood of any of its points. If a sequence $\left\{P_{n}\right\}$ with $P_{n} \in \pi_{n, \theta}$ converges uniformly on $S_{\theta}$ as $n \rightarrow \infty$ then $\left\{P_{n}(w)\right\}$ converges to 0 for every $w \in K \backslash S_{\theta}$.

Proof. Suppose that $P_{n} \in \pi_{n, \theta}$ with $P_{n} \rightarrow f_{0}$ uniformly on $S_{\theta}$ as $n \rightarrow \infty$ and let $w=\left(w_{1}, \ldots, w_{N}\right) \in K \backslash S_{\theta}$. Define $p(z)=p\left(z_{1}, \ldots, z_{N}\right)=\left(z_{1}^{2}+\cdots\right.$ $\left.+z_{N}^{2}\right)^{\gamma}\left(\left(z_{1}-w_{1}\right)^{2}+\cdots+\left(z_{N}-w_{N}\right)^{2}\right)^{\beta}$ where $\gamma$ and $\beta$ are positive integers such that $\gamma /(\gamma+\beta)>\theta$. With this choice, $p$ is a $\theta$-incomplete polynomial. Furthermore, since $K \subset \mathbb{R}^{N}, p$ only vanishes on $K$ at $w$ and at the origin. 
Now, $S_{\theta}$ and

$$
\begin{array}{r}
A=\left\{f \in C\left[S_{\theta}\right]: \exists Q_{n} \in \pi_{n, \theta} \text { with } Q_{n} \rightarrow f \text { uniformly as } n \rightarrow \infty\right. \\
\text { and } \left.Q_{n}(w)=0\right\}
\end{array}
$$

satisfy the hypotheses of the Stone-Weierstrass theorem.

Hence $p(z) f_{0}(z) \in A$ iff $p(z) f_{0}(z) \equiv 0$ on $Z(A)$ iff $f_{0}(z) \equiv 0$ on $Z(A)$ iff $f_{0}(z) \in A$.

The second equivalence holds since $Z(A) \subset S_{\theta}$ and $f_{0}(0)=0$. Hence, $f_{0} \in A$. It follows that there exist $Q_{n} \in \pi_{n, \theta}$ with $Q_{n} \rightarrow f_{0}$ on $S_{\theta}$ uniformly as $n \rightarrow \infty$, with $Q_{n}(w)=0$. Thus $\left|P_{n}(w)\right| \leq\left\|Q_{n}-P_{n}\right\|_{K}=\left\|Q_{n}-P_{n}\right\|_{K}^{*}=$ $\left\|Q_{n}-P_{n}\right\|_{S_{\theta}}=\left\|Q_{n}-P_{n}\right\|_{S_{\theta}}$. Letting $n \rightarrow \infty$ we get $\left|P_{n}(w)\right| \rightarrow 0$.

Corollary 4.12. $K \backslash S_{\theta} \subset Z_{\theta}$.

Proof. Any sequence $P_{n} \in \pi_{n, \theta}$ converging uniformly on $K$ satisfies the hypotheses of the previous theorem.

THEOREM 4.13. If $K \subset \mathbb{R}^{N}$ is an intersection of compact sections and is non-pluripolar in a neighbourhood of any of its points then

$$
S_{\theta}=\overline{K \backslash \theta^{2} K} \cup\{0\} .
$$

Proof. From [2] we have $Z_{\theta}=\theta^{2} K$. Moreover the inclusion $K \backslash S_{\theta} \subset Z_{\theta}$ gives $K \backslash \theta^{2} K \subset S_{\theta}$. We also know that $\{0\} \subset S_{\theta}$. Now if $\|P\|_{K \backslash \theta^{2} K}^{*}=\|P\|_{K}^{*}$ for all $\theta$-incomplete polynomials $P$ then by Theorem $4.8, S_{\theta} \subset \overline{K \backslash \theta^{2} K}$ $\cup\{0\}$ and we are done. Otherwise we have $\|P\|_{K \backslash \theta^{2} K}^{*}<\|P\|_{K}^{*}$ for some $\theta$-incomplete polynomial $P$. Consequently, $\|P\|_{K \backslash \theta^{2} K}<\|P\|_{K}$. In this case take $q \in \theta^{2} K$ with $|P(q)|=\|P\|_{K}$. Because $K$ is an intersection of compact sections, it is starlike with respect to the origin, so restricting to the line through the origin and $q$ we get a one-variable $\theta$-incomplete polynomial $p$ with $|p(q)|>\|p\|_{\left[\theta^{2}, 1\right]}$, where $q<\theta^{2}$. This contradicts the fact that $\|p\|_{\left[\theta^{2}, 1\right]}=\|p\|_{[0,1]}$ for all $\theta$-incomplete polynomials $p$.

\section{References}

[1] E. Bedford and B. A. Taylor, Plurisubharmonic functions with logarithmic singularities, Ann. Inst. Fourier (Grenoble) 38 (1988), no. 4, 133-171.

[2] M. M. Branker, Approximation by weighted polynomials in $\mathbb{R}^{k}$, Ann. Polon. Math. 85 (2005), 261-279.

[3] J.-P. Demailly, Mesures de Monge-Ampère et mesures pluriharmoniques, Math. Z. 194 (1987), 519-564.

[4] M. von Golitschek, Approximation by incomplete polynomials, J. Approx. Theory 28 (1980), 155-160.

[5] M. Klimek, Pluripotential Theory, Clarendon Press, Oxford, 1991.

[6] A. B. J. Kuijlaars, A note on weighted polynomial approximation with varying weights, J. Approx. Theory 87 (1996), 112-115. 
[7] G. G. Lorentz, Problems for incomplete polynomials, in: Approximation Theory III (Austin, TX, 1980), Academic Press, New York, 1980, 41-73.

[8] E. B. Saff and V. Totik, Logarithmic Potentials with External Fields, Grundlehren Math. Wiss. 316, Appendix B by T. Bloom, Springer, Berlin, 1997.

[9] E. B. Saff and R. S. Varga, The sharpness of Lorentz's theorem on incomplete polynomials, Trans. Amer. Math. Soc. 249 (1979), 163-186.

[10] J. Siciak, A remark on Tchebysheff polynomials in $\mathbb{C}^{N}$, Univ. Iagel. Acta Math. 35 (1997), 37-45.

Department of Mathematics

University of Toronto

Toronto, Ontario

M5S 2E4, Canada

E-mail: calaghan@math.toronto.edu

Received 2.3.2006

and in final form 20.7.2006 